\title{
Program library for DECtalk text-to-speech system
}

\author{
SIMON LOCK and CHE KAN LEONG \\ University of Saskatchewan, Saskatoon, Saskatchewan, Canada
}

\begin{abstract}
The DECtalk text-to-speech conversion system offers promise for both basic and applied research. This paper is a report on a program library for the system that utilizes the routines by Edwards (1987). The library contains a number of useful Pascal routines which simplify the basic control of DECtalk. The demonstration program that we have devised should assist users of the system.
\end{abstract}

In recent years, there has been considerable interest in text-to-speech algorithms and in the use of this technology in the study of computer-mediated reading instruction. The MIT Research Laboratory of Electronics, among others, has led in the development of the MITalk-79 system of text-to-speech conversion (see Allen, Hunnicutt, \& Klatt, 1987, for details). The Digital Corporation has also provided a sophisticated DECtalk text-to-speech conversion system.

In general, the transformation of strings of ASCII characters into speech is based on an analysis-by-synthesis procedure. The computer system first analyzes the unrestricted text to extract the underlying phonemic, morphological, and syntactic forms. The system then uses this abstract linguistic representation as the input to the speechsynthesis component, which also entails segmental phonological adjustments such as utterance timing and pitch contour for high-quality speech. The voice output devices utilizing computer speech have been evaluated, and the DECtalk computer system has been shown to produce highly intelligible synthetic speech (Greene, Logan, \& Pisoni, 1986).

Several researchers have investigated the use of computer speech in computer-mediated reading. McConkie and Zola (1985) reported on their Computer-Aided Reading System, which displays text and provides instantaneous speech feedback of difficult words when they are "touched" on the screen. Olofsson (1988) discusses some Swedish text-to-speech systems that convert subword units into speech sounds to train disabled readers. Directly pertaining to DECtalk, the system has been used with considerable success by Olson and his colleagues to give speech feedback for word recognition and reading comprehension with reading-disabled children (see Olson,

The work reported here was facilitated by the Social Sciences and Humanities Research Council of Canada through the award of SSHRCC Research Grant 410-87-0058 to Che Kan Leong. We are grateful to SSHRCC, the University of Saskatchewan, and Digital Equipment of Canada Ltd. for their assistance. We also thank the editor and the anonymous reviewers for their insightful comments. Address correspondence to Che Kan Leong, Department for the Education of Exceptional Children, University of Saskatchewan, Saskatoon, Saskatchewan S7N 0W0, Canada.
Foltz, \& Wise, 1986; Olson \& Wise, 1987). We have also been experimenting with DECtalk to provide immediate on-line and speech feedback to older readers during prose comprehension.

In this paper, we describe a library of Turbo Pascal routines that can control the basic operations of DECtalk on an IBM PC-compatible computer. These routines are compiled with the Turbo Pascal compiler (version 3.01A) on a Digital VAXmate computer, which is fully compatible with the IBM AT. The objectives of the library are twofold. First, it contains a number of useful routines that simplify the technical details of controlling the basic features of DECtalk. Second, it contains a general displaying routine that can present a given text passage in many ways. The listing of the library is shown in Appendix A. A demonstration program is given in Appendix B, in order to illustrate the use of the routines in the library.

\section{Operating Modes of DECtalk}

DECtalk has three operating modes: the setup mode, the on-line mode, and the off-line mode (see DECtalk Owner's Manual for detail). In the setup mode, DECtalk allows the user to set various internal parameters such as line speed, data format, trace flags, and the like. In the on-line mode, DECtalk communicates directly with a host computer using escape sequences. The escape sequences are sequences of characters that start with an escape (ESC) character, followed by a string of ASCI characters. They are commands that control the operating characteristics of DECtalk. In the off-line mode, DECtalk acts as a text-to-speech system that accepts commands from the local terminal. In terms of control, the on-line mode is better than the off-line mode, because it provides a more extensive set of commands for the programmer. For example, in measuring the speaking rate of DECtalk, one needs to know exactly when DECtalk finishes speaking: the on-line mode has an escape command that requests DECtalk to send a signal back once the speaking is finished.

\section{Communicating with DECtalk}

DECtalk is physically linked with the host computer by a serial cable, through which signals are transmitted 
according to the commonly used RS232 protocol. This protocol defines a mutual agreement between two communicating devices; without it, messages sent from one device will be misinterpreted by the other. In order to facilitate communication between DECtalk and the IBM PC, we must program both of them to transmit data at the same rate and with the same format. We have set DECtalk to transmit data at a rate of 9,600 baud, with no parity checking. This protocol can be specified in the setup mode, with the following commands:

\section{SETUP > SET HOST SPEED 9600 SETUP $>$ SET HOST FORMAT NONE}

The IBM PC is programmed to transmit data with the same protocol. To program the serial port of the IBM PC with Turbo Pascal is quite common for many applications, but it cannot be done with just a few lines of codes. There are many ready-to-use routines commercially available to do such a task. In particular, we have chosen the routines in Edwards (1987), who discusses the programming details of data communication on the IBM PC. The routines that we use are contained in the modules GENERAL.INC, INTER.INC, and DATACOMM.INC. In the remaining part of this paper, we will describe the details of the DECtalk library DECTALK.INC, which is listed in Appendix $\mathbf{A}$.

\section{Using the DECtalk Library}

Certain lines of codes must be included in any program that uses the DECtalk library. First, since the DECtalk library DECTALK.INC makes use of the data communication routines in module DATACOMM.INC, which in turn uses routines in modules GENERAL.INC and INTER.INC, the following lines must be included in all programs that use the library:

\section{\{\$I GENERAL.INC \\ \{\$I INTER.INC\} \\ \{\$I DATACOMM.INC\} \\ \{\$I DECTALK.INC\}}

Second, the serial port of the IBM PC must be set up before the program sends any command to DECtalk, and it must be reset after the program finishes the communication with DECtalk. The setting of the serial port on the IBM PC can be accomplished by calling the procedure RS_Initialize() in module DATACOMM.INC. To set the transmission rate at 9,600 baud with no parity bit, the following call is used:

\section{RS_Initialize(1,B9600,None, 1,8);}

Similarly, there are a number of DECtalk escape sequences that must be defined before DECtalk is used. An initialization procedure, DECtalk Initialize(), is written to perform such tasks. This procedure should be called before the program sends any escape sequence to DECtalk:

\section{DECtalk_Initialize;}

To reset the communication port, the RS_Cleanup() procedure in the DATACOMM.INC module is used. This procedure call should be put after the program finishes communicating with DECtalk:

\section{RS_Cleanup;}

The routines in the DECtalk library can be classified into three types: speaking, displaying, and miscellaneous routines. The speaking routines are used to control DECtalk to speak a given text in a variety of ways. The displaying routine, on the other hand, is used to control the screen display of a given text in different ways. The miscellaneous routines control other useful features of DECtalk, such as changing its voice, its speaking rate, and many others.

\section{Speaking Routines in DECTALK.INC}

The basic routine of communication is to send a character to DECtalk. The procedure Send() is written to accomplish this task. After the program has initialized the serial port using RS__nitialize(), an 8-bit data can be sent to DECtalk through the procedure Send(), which is a modified version of the procedure RS232_Out() in module DATACOMM.INC.

A sequence of characters, followed by a carriage return, can be sent to DECtalk with the procedure Speak(). This procedure makes use of the previous basic routine Send().

If one wants to know when DECtalk finishes speaking a message, the procedure SyncSpeak() can be used to send the message and detect the end of speaking with Get_SyncSignal(). To synchronize with DECtalk, one first calls SyncSpeak() with the message. While DECtalk is speaking the message, the computer can do other tasks, such as print it. When DECtalk finishes speaking the message, the Boolean function RS232__Avail() will return true, indicating that DECtalk has sent some messages back. The main responsibility of the procedure Get__SyncSignal() is to detect a synchronization signal DT__SYNCSIGNAL. It uses another procedure called Get_Response to get the response from DECtalk. The procedure Get_Response puts the receiving message in a global string variable called Input_String. The program structure for synchronization is as follows:

$$
\begin{gathered}
\text { SyncSpeak(Message); } \\
: \\
\text { \{ Do some tasks }\} \\
\quad: \\
\text { Get_SyncSignal; }
\end{gathered}
$$

If the computer finishes the tasks earlier than DECtalk finishes speaking the message, it will be held up in the busy-wait loop of Get_SyncSignal(). In any case, DECtalk is synchronized once Get_SyncSignal() returns to the main program. 


\section{Displaying Routines in DECTALK.INC}

One simple way to interface on-line and speech output is to use the standard WriteLn() function to print the passage, but this may not be acceptable because the text presentation rate is so fast. An alternative is to delay the displaying of characters by using the Delay() function. The advantage of this approach is that characters are displayed on the screen very smoothly. There are other presentation methods, such as delaying between words, delaying between phrases, and delaying between sentences. In the procedure Display(), all of these different presentation methods are included. There are five parameters in this procedure. The first is the text message (maximum 255 characters) that is to be displayed on the screen. The second parameter specifies the delay time (in milliseconds, approximately) between characters. Similarly, the third, the fourth, and the fifth parameters specify the delay time between words, phrases, and sentences, respectively. The procedure considers each character one by one and determines which delay time to use according to the nature of the character. If the character is a period, an exclamation mark, or a question mark, then the procedure delays the processing by means of the fifth parameter. If the character is a comma, a semicolon, or a colon, then it treats the punctuation as a phrase delimiter. It will then use the delay time specified by the fourth parameter. These definitions of delimiters are declared at the beginning of the DECtalk library. Although this general displaying routine is general enough to allow different delays between linguistic frames of different units, it is not intelligent enough to recognize some of the special forms in English such as abbreviations. More work remains to be done to improve this procedure. However, the procedure in its current implementation can be used for a wide variety of tasks.

\section{Miscellaneous Routines in DECTALK.INC}

Many other DECtalk features, such as the speaking rate and voice are also programmable. The default speaking rate is approximately $180 \mathrm{wpm}$, but one can vary the rate from 120 to $250 \mathrm{wpm}$ with the procedure Speaking_Rate(). To set DECtalk to speak at 200 wpm, one calls Speaking_Rate(200). The default pauses of 160 msec after commas and $640 \mathrm{msec}$ between sentences can be lengthened with the procedures Comma_Pause() and Period_Pause(). Both procedures accept one argument, which sets the additional delay after the comma and period. To set the delay after a comma at $200 \mathrm{msec}$, one calls Comma_Pause(40). Similarly, if one wants the delay between sentences to be $740 \mathrm{msec}$, one calls Period_Pause(100). However, when one uses these procedures to lengthen the pauses after commas or periods, the speaking rate of DECtalk will be affected.

Another interesting feature is to change the voice of DECtalk. DECtalk has seven built-in voices: a standard male voice, a standard female voice, a deep male voice, an older male voice, a child's voice, a deep female voice, and a light female voice. The procedure Change_Voice() selects the voice. The default voice is the standard male "Perfect Paul." For DECtalk to speak in a light female voice, one calls Change__Voice(7).

The pronunciations produced by DECtalk have high fidelity in most cases, but they are not perfect. Because there are so many exceptions to the rules of English pronunciation, the built-in dictionary of DECtalk contains only the most common exceptions. DECtalk has another user dictionary that can be maintained in order to modify the pronunciations of certain words. The best way to do this is to try out the pronunciation via the off-line mode, with the local terminal. If a desirable pronunciation can be found, the function Update__Dictionary() can be used to update the user's dictionary. This function accepts the word to be changed as the first argument and the new pronunciation as the second argument. It will then return a character sent back from DECtalk, indicating whether the update is successful or not. A " 0 " character indicates that DECtalk has accepted the word successfully. If the returned character is " 1 ," then it means that DECtalk has no room to store the given word. If a " 2 "' character is returned, the entry is too long (i.e., longer than 256 characters).

\section{Demonstration Program}

This demonstration program was devised to (1) illustrate the program structure for using the DECtalk library DECTALK.INC, and (2) demonstrate the selection of different parameters in the displaying routine. The program defines a passage of text at the beginning and controls DECtalk to speak each line of the passage with different voices. The displaying speed of each line also varies from fast to slow, and the synchronization structure is used to coordinate the computer with DECtalk. This demonstration will show that the computer displays the first few lines faster than DECtalk. However, as the delay times between linguistic frames of different sizes increase, DECtalk will speak faster than the text display in the last few lines. Synchrony for on-line display and speech output is achieved at the end of each line.

\section{Conclusion}

The DECtalk text-to-speech computer system offers promise for basic research on the mechanisms of speech feedback of unrestricted text, and for applied research on component analysis and remediation of reading.

\section{REFERENCES}

Allen, J., Hunnicutt, M. S., \& Klatt, D. (1987). From text to speech: The MITalk system. Cambridge, England: Cambridge University Press.

Edwards, C. C. (1987). Advanced techniques in Turbo Pascal. San Francisco: Sybex. 
Greene, B. G., Logan, J. S., \& Pisoni, D. B. (1986). Perception of synthetic speech produced automatically by rule: Intelligibility of eight text-to-speech systems. Behavior Research Methods, Instruments, \& Computers, 18, 100-107.

McConkie, G. W., Zola, D. (1985, April). Computer-aided reading: An environment for developmental research. Paper presented at the meeting of the Society for Research in Child Development, Toronto, Canada.

OLOFsSON, $\dot{A}$. (1988). Phonemic awareness and the use of computer speech in reading remediation: Theoretical background. Fonetiks, 1 , 15-27.

Olson, R., Foltz, G., \& Wise, B. (1986). Reading instruction and remediation with the aid of computer speech. Behavior Research Methods, Instruments, \& Computers, 18, 93-99.

Olson, R., \& Wise, B. (1987). Computer speech in reading instruction. In D. Reinking (Ed.), Computers and reading: Issues for theory and practice (pp. 156-177). New York: Teachers College Press, Columbia University.

\section{APPENDIX A}

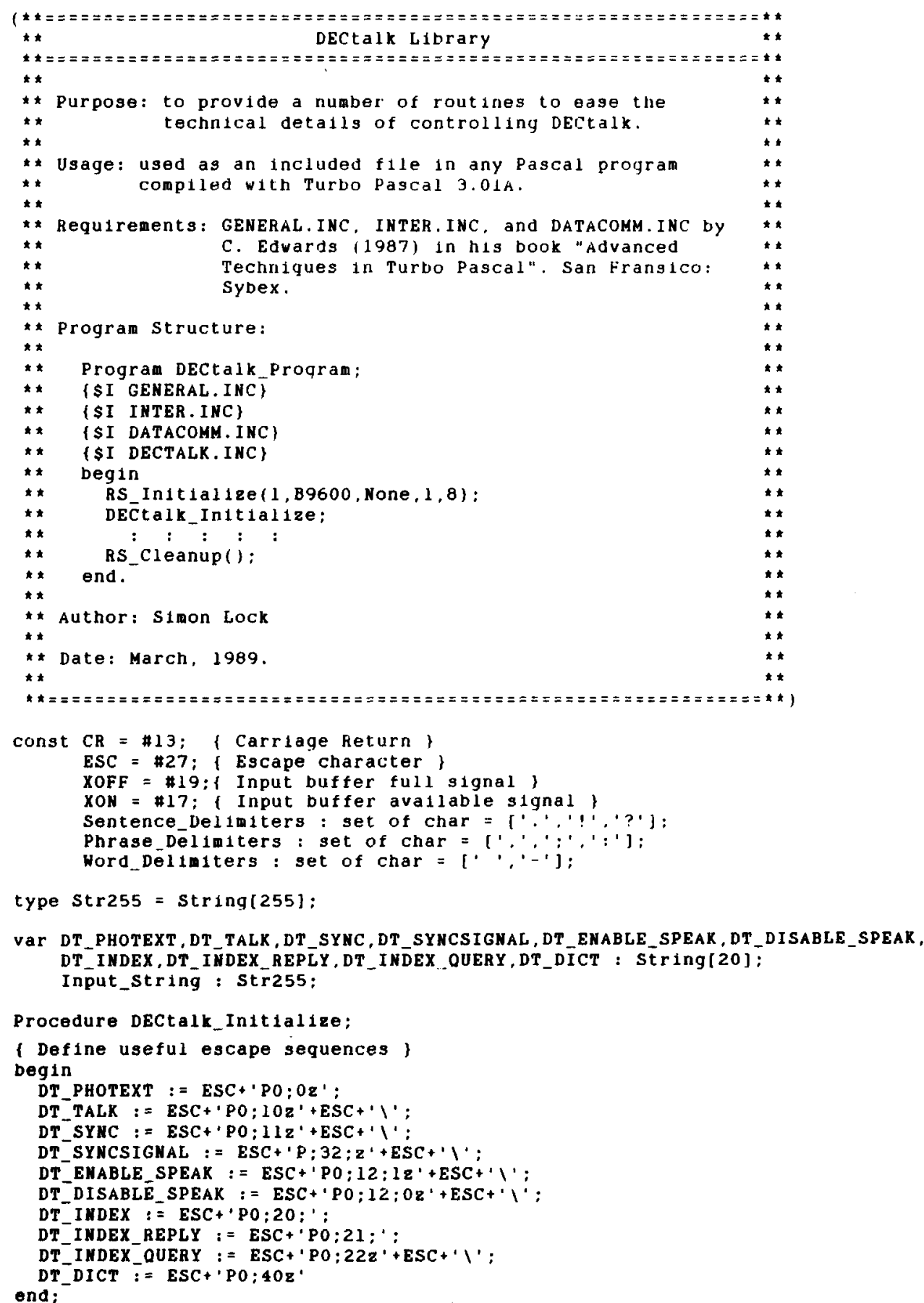




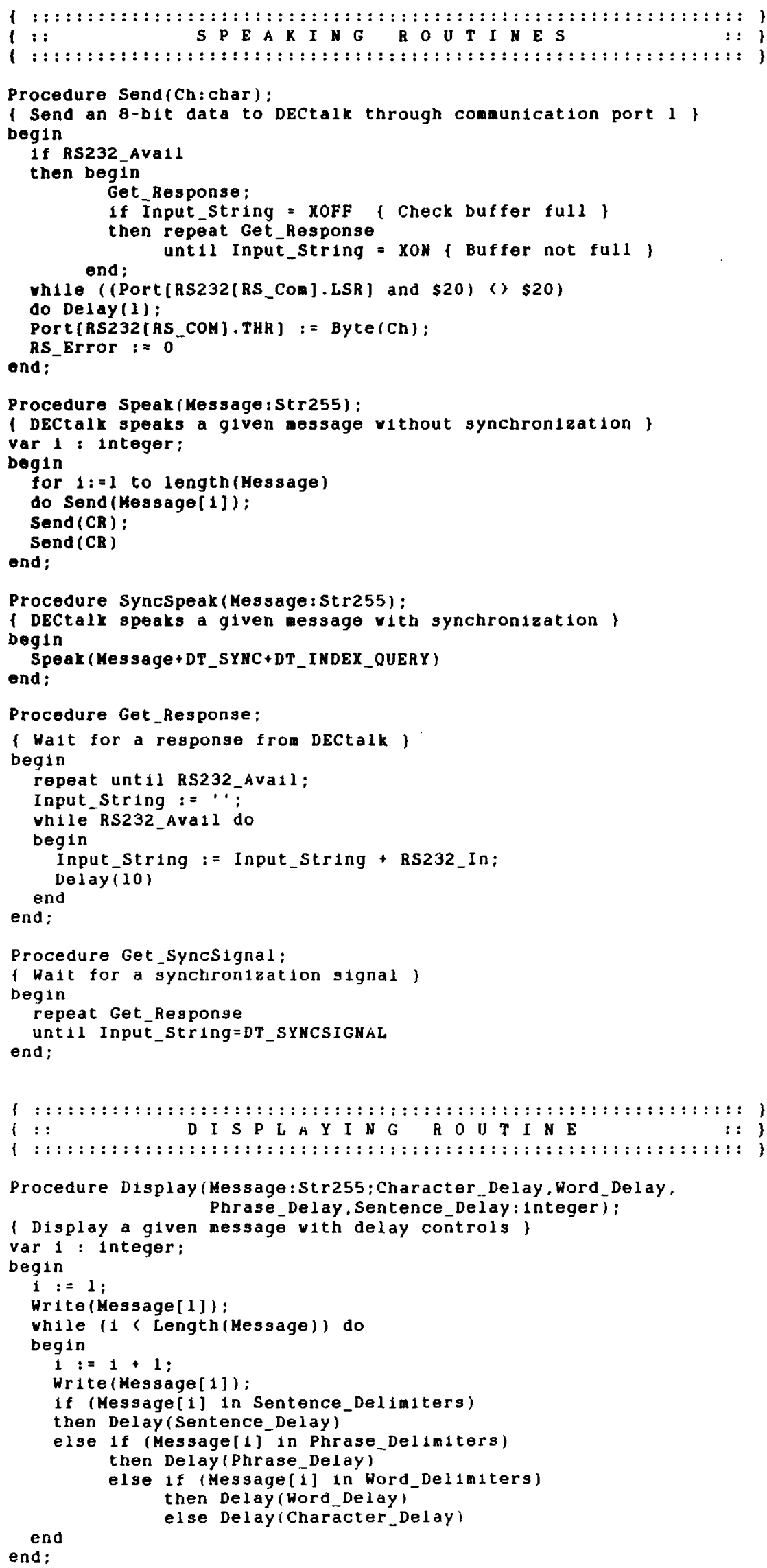




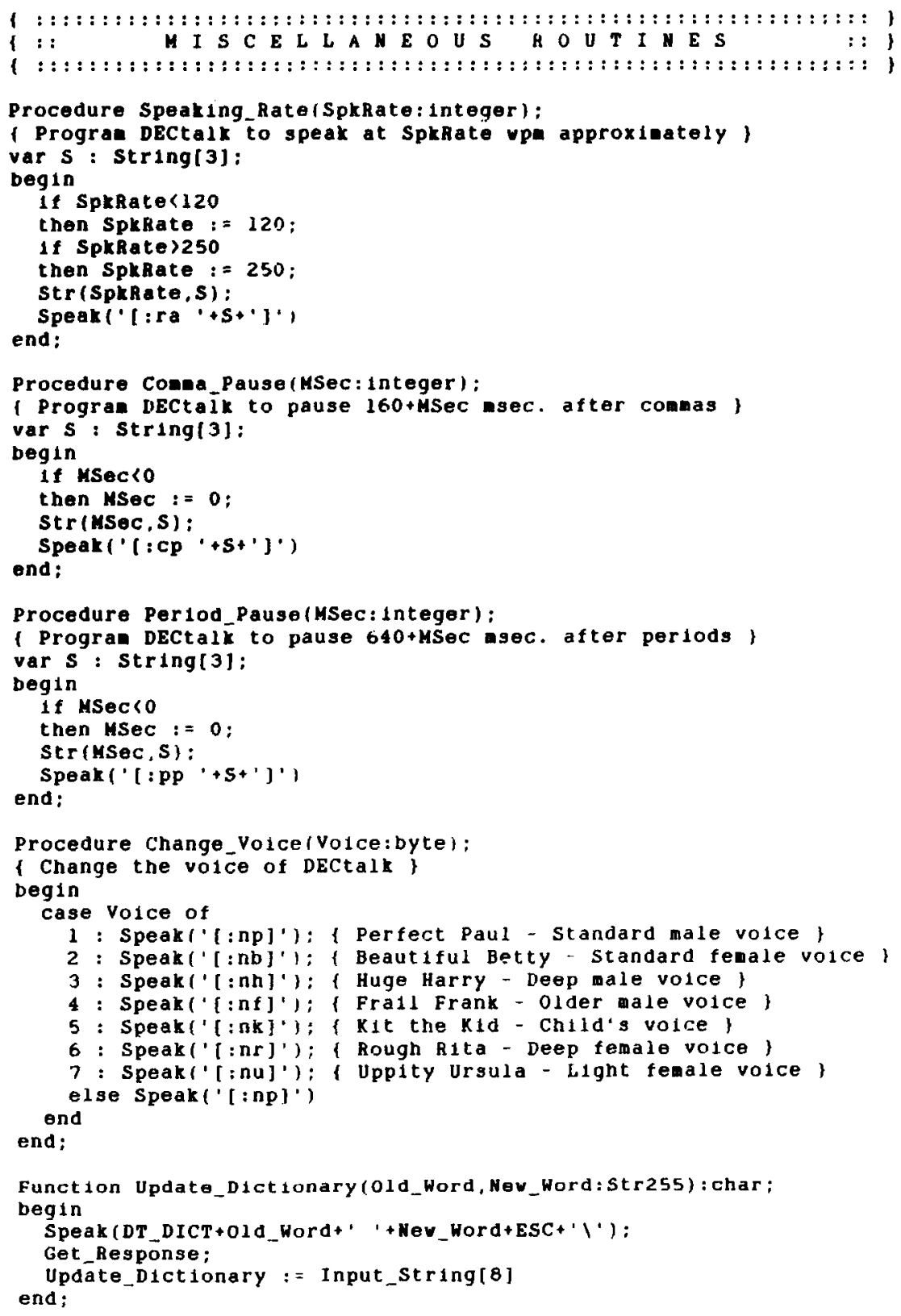

\section{APPENDIX B}

Program DECtalk_Demongtration_Program;

\{\$I GENERAL. INC \}

\{\$I INTER . INC \}

$\{\$ I$ DATACOMM. INC $\}$

\{\$I DECTALK. INC \}

const Passage: array $[1, .8]$ of $5 t r 255=$

('In this paper, we describe a library of Turbo Pascal routines'.

'to control the basic operations of DECtalk on an IBM PC conpatible'.

'computer. These routines are complied vith the Turbo Pascal'

'compiler on a Vaxmate computer. The objectives of the library are'.

'twofold. Firstiy, it contains a number of useful routines to'.

'simplify the technical detalis for controlling the basic features'.

' of DECtalk. Secondly, it contains a general displaying routine'.

'which can present a given text passage in many vays.'):

var 1 : Integer: 


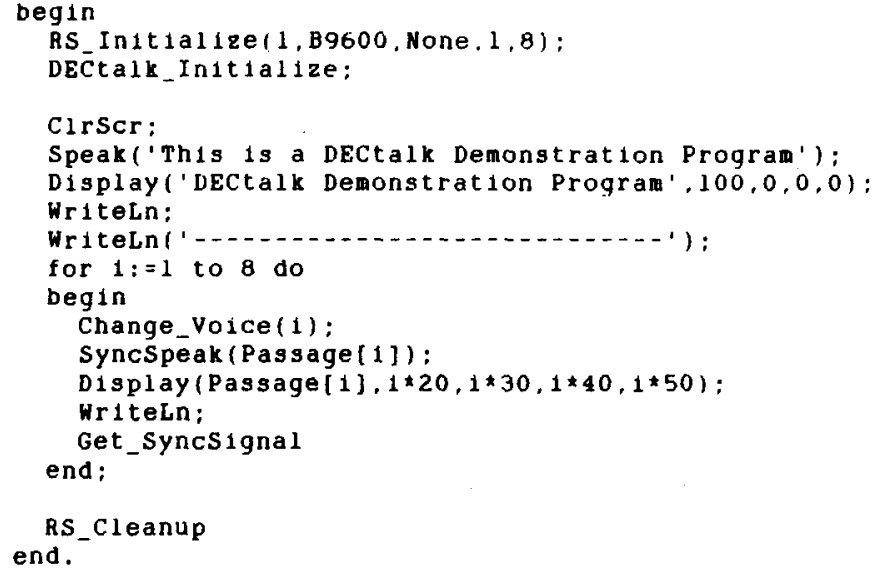

(Manuscript received December 22, 1988;

revision accepted for publication March 30, 1989.) 